\title{
Strange New Magnetics from the Sloan Digital Sky Survey
}

\author{
Paula Szkody \\ Dept. of Astronomy, University of Washington, Box 351580, Seattle, \\ WA 98195
}

\begin{abstract}
The first two years of the Sloan Digital Sky Survey have discovered over 50 new cataclysmic variables, of which four are confirmed Polars, two others are likely candidates and several others are good candidates for Intermediate Polars or SW Sex stars. Several systems have unusual characteristics, including large amplitude, narrow cyclotron humps indicating a very low temperature shock on a cool white dwarf, or very deep eclipses. Although SDSS is finding a variety of systems, it is ideal for picking up the low mass transfer rate magnetic systems missed in Xray surveys and in past optical surveys with brighter limits. Thus, it is providing us with a clearer picture of the range of conditions existing in magnetics CVs.
\end{abstract}

\section{Introduction}

The Sloan Digital Sky Survey (SDSS) is a five year project designed to obtain photometry of 10,000 square degrees of the sky in 5 filters $(u, g, r, i, z)$ to fainter than 20th magnitude, using a $2.5 \mathrm{~m}$ telescope at Apache Point Observatory (APO). A software pipeline then selects objects by color for multi-fiber spectroscopy on the same telescope, producing blue and red spectra that cover $3900-9200 \AA$ at a resolution near $3 \AA$. Details of the project and equipment are described in Gunn et al. (1998), Fukugita et al. (1996), Lupton, Gunn \& Szalay (1999), Lupton et al. (2001) and York et al. (2000) as well as the SDSS web page at www.sdss.org.

While the main goal of the SDSS project is to obtain redshifts for galaxies and quasars, the images and spectra also contain many stellar objects. A stars working group is allocated about 10 spectral fibers (out of 640 per 3 degree field) for stellar goals. Among these, one fiber is allowed for cataclysmic variables (CVs). Since CVs have a very broad range of colors (depending on whether the accretion disk, accretion column, or the underlying stars contribute most of the light), various attempts to isolate them by color proved disappointing. The test year showed the best way to eliminate normal stars and quasars was to set the color selection to pick up objects that were both very blue and very red (WD+M pairs), which could be pre-CVs or low mass transfer CVs. However, by far, the largest number of spectra of CVs are produced by the quasar color selections, as the quasar color loci fall within those for CVs and they have a large number of fibers. In addition, the serendipity working group also finds some CVs that have unusual colors. In the end, all spectra produced by SDSS are manually 
searched for objects that look like CVs i.e. that have broad Balmer and Helium emission lines at zero red-shift.

The commissioning year of SDSS (released as the Early Data Release or EDR; Stoughton et al. 2002) showed that the survey is able to find many new CVs. In this year (through December 2000), 22 spectra of CVs were obtained, with 19 being new systems (Szkody et al. 2002; Paper 1). The following year (through December 2001) includes the spectra of 42 additional CVs (Szkody et al. 2003; Paper 2), with about 35 being new discoveries. Among these systems, about $25 \%$ show the signatures of magnetic systems i.e. strong HeII 4686 emission and/or multiple components in the Balmer lines (some examples are shown in Figure 1). The newly discovered CVs are being followed up with the $3.5 \mathrm{~m}$ telescope at APO, using the Double Imaging Spectrograph to obtain timeresolved blue and red spectra at $3 \AA$ resolution and with the UW $0.76 \mathrm{~m}$ telescope at Manastash Ridge Observatory and the USNO telescope to obtain light curves. The polarimeter at Steward Observatory is also being used to obtain circular polarization measurements of the polar candidates. Some of our available results are described below.

\section{Polars}

At the current time, there are 4 confirmed (from the presence of circular polarization) polars and several other candidates (based on the strength of HeII and the structure in the emission lines). The confirmed systems include SDSS0155 (Paper 1), SDSS1553 and SDSS1324 (Szkody et al. 2003b) and SDSS1700 (Paper 2). The latter 3 systems are shown in Figure 1, while SDSS0155 was so bright that it is saturated in the SDSS spectrum (the APO followup spectra are shown in Paper 1).

SDSS1553 and SDSS1324 are among the most unusual systems of the group. They have very extreme cyclotron humps together with $\mathrm{TiO}$ bands from the secondary star. As described in more detail in the papers by Schmidt and Ferrario et al. in this volume, these 2 systems are modelled with the lowest specific accretion rates onto the coolest white dwarfs yet known in a CV. These kinds of systems are difficult to search for by color as they appear in odd locations in SDSS color-color plots, since their colors are primarily determined by the locations of the cyclotron humps in the spectrum. Time-resolved spectroscopy (Szkody et al. 2003b) shows that these humps change in amplitude during the orbital cycle. Systems such as these can be selected serendipitously by SDSS for spectral fibers if the hump amplitudes place them within the quasar color cuts at the time when the SDSS image is taken. However, even with the resulting SDSS spectra, the low signal-to-noise levels for these faint sources make it difficult to distinguish extreme polar systems of this type from quasars that show only one line. Thus, the positive confirmation of these objects as polars must come from spectropolarimetry, which reveals large circular polarization at the wavelengths corresponding to the cyclotron features. At the current time, there are 2 other candidates waiting for confirmation. While these are not the first polars to show large amplitude cyclotron features (see Schwope, Schwarz \& Greiner 1999; Reimers \& Hagen 2000), they are the most extreme of this type. In addition, SDSS seems to be picking up more of them (previous to SDSS, about $5 \%$ of 

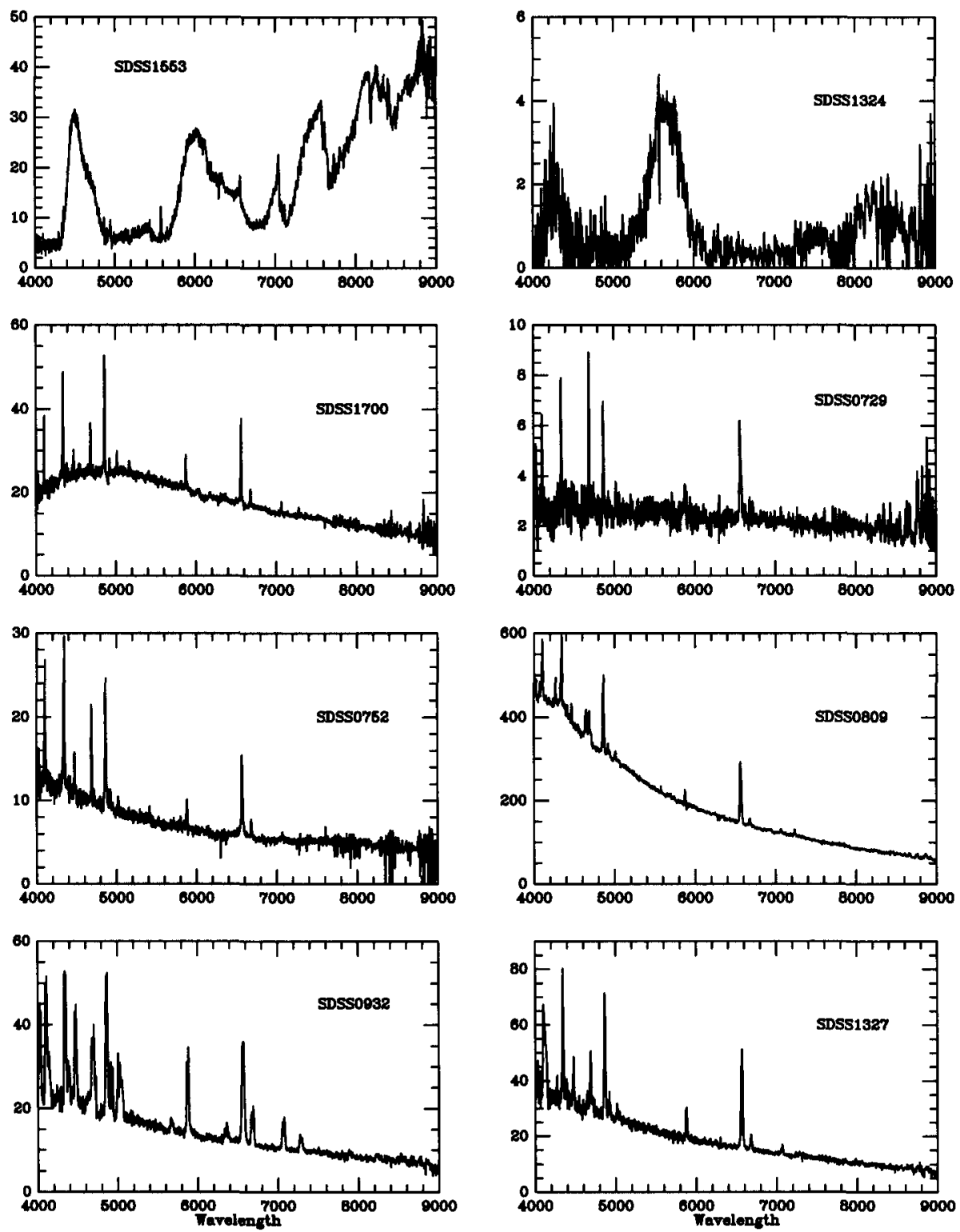

Figure 1. Sample SDSS spectra showing the presence of strong HeII which is an indication of a possible magnetic CV. The $y$-axis is flux $\times 10^{-17} \mathrm{ergs} \mathrm{cm}^{-2} \mathrm{~s}^{-1} \AA^{-1}$. 
polars were known to be in this low accretion regime, whereas $20-40 \%$ of the new polars discovered with SDSS are of this type). Thus, this low accretion regime may represent a larger component of the magnetic $\mathrm{CV}$ population than previously thought.

Another unusual system is SDSS0155 (Paper 1) which has an 87 min period and has extremely deep ( 6 magnitude) eclipses, indicating an unusually bright accretion area. SDSS1700 (Figure 1) is fairly normal in appearance in the SDSS spectrum. It is also highly modulated during its $115 \mathrm{~min}$ orbit, displaying a $2.5 \mathrm{mag}$ variation in $\mathrm{V}$ photometry as well as a variation in circular polarization from 3-27\% (Paper 2). Other polar candidates (such as SDSS0729 and SDSS0752 shown in Figure 1) remain to be confirmed. XMM-Newton observations have recently been obtained for SDSS0729 and SDSS0752 and will soon be accomplished for SDSS0155, which should help to establish the accretion conditions in these systems.

\section{Intermediate Polars/SW Sex Stars}

Since Rodriguez-Gil et al. (2001) first showed that some of the SW Sex stars exhibit low values of circular polarization, it is possible that SW Sex systems are synonymous with intermediate polars. Thus, we will include them all in this category although each type has different signatures that result in their classification as either IPs or SW Sex stars. The intermediate polars are generally found in a range of periods above the period gap while the SW Sex stars are primarily concentrated in the $3-4 \mathrm{hr}$ period regime. The intermediate polars usually are distinguished by photometry that shows the spin period of the white dwarf (largest amplitude in X-rays) while the SW Sex stars are generally characterized by spectroscopy that shows the presence of HeII with a strength $\geq \mathrm{H} \beta$ and single-peaked Balmer emission except for deep absorption generally around phases 0.5-0.7. In addition, many SW Sex stars show a strong CN blend at $4640 \AA$. Thus, the SDSS candidates generally have to be followed with photometry to identify spin/orbital periods and with time-resolved spectroscopy to determine the spectral absorption features.

Figure 1 shows some typical candidates. SDSS1327 is a typical high-inclination eclipsing system with an orbital period of $3.2 \mathrm{hrs}$ (Wolfe et al. 2003) while SDSS0809 is a low inclination system with a period near $2.4 \mathrm{hrs}$. Both show absorption features that link them to SW Sex stars. The most peculiar members of this group are the ones showing strong doubled lines along with strong HeII. The first one known of this type in the SDSS spectra was identified by Bond et al. (2002) as a radio source. Figure 1 shows a system with similar optical spectral characteristics (SDSS0932).

\section{Summary}

The first 2 years (commissioning year plus first full year) of the SDSS project have obtained spectra of over $60 \mathrm{CVs}$. Over 50 of these are new discoveries and about $25 \%$ are likely candidates for magnetic polars or intermediate polars/SW Sex stars. These results indicate a great variety of systems are being discovered with this survey, including systems with both high and low accretion rates and 
with a variety of inclinations. At the end of the 5 years, we expect to double the number of currently known magnetic systems. One of the most noteworthy results so far is the large number of very low accretion rate polars compared to polars discovered from X-ray surveys. It is apparent that many magnetics may have buried shocks, with no apparent X-ray emission and the general population may be quite different from what past surveys have indicated. Observers (especially those with large telescopes) are encouraged to obtain further followup data on the many systems that are being found.

Acknowledgments. This work was partially funded with NSF grant AST0205875 . We gratefully acknowledge the SDSS collaborators who participated in identifying and working on these magnetic CVs, particularly Scott Anderson, Oliver Fraser, James Frith, Patrick Hall, Arne Henden, Brandon Lawton, Gary Schmidt and Michael Wolfe. Funding for the creation and distribution of the SDSS Archive has been provided by the Alfred P. Sloan Foundation, the Participating Institutions, the National Aeronautics and Space Administration, the National Science Foundation, the U.S. Department of Energy, the Japanese Monbukagakusho, and the Max Planck Society. The SDSS Web site is http://www.sdss.org/. The SDSS is managed by the Astrophysical Research Consortium (ARC) for the Participating Institutions. The Participating Institutions are The University of Chicago, Fermilab, the Institute for Advanced Study, the Japan Participation Group, The Johns Hopkins University, Los Alamos National Laboratory, the Max-Planck-Institute for Astronomy (MPIA), the MaxPlanck-Institute for Astrophysics (MPA), New Mexico State University, University of Pittsburgh, Princeton University, the United States Naval Observatory, and the University of Washington.

\section{References}

Bond, H. E., White, R. L., Becker, R. H., \& O'Brien, M. S. 2002, PASP, 114, 1359

Fukugita, M., Ichikawa, T., Gunn, J. E., Doi, M., Shimasaku, K., \& Schneider, D. P. 1996, AJ, 111, 1748

Gunn, J. E., Carr, M., Rockosi, C. (+37 co-authors) 1998, AJ, 116, 3040

Lupton, R. H., Gunn, J. E., \& Szalay, A. 1999, AJ, 118, 1406

Lupton, R. H., Gunn, J. E., Ivezic, Z., Knapp, G. R., Kent, S. M., \& Yasuda, N. 2002, in ASP Conf. Ser. Vol. 238, 269

Reimers, D., \& Hagen, H. J. 2000, A\&A, 358, L45

Rodriguez-Gil, P., Casares, J., Martinez-Pais, I. G., Hakala, P., \& Steeghs, D. 2001, ApJ, 548, 49L

Schwope, A. D., Schwarz, R., \& Greiner, J. 1999, A\&A, 348, 861

Stoughton, C., Lupton, R. H., Bernardi, M. (+189 co-authors) 2002, AJ, 123, 485

Szkody, P., Anderson, S. F., Agüeros (+27 co-authors) 2002, AJ, 123, 430 (Paper 1)

Szkody, P. et al. 2003, in prep (Paper 2) 
Szkody, P., Anderson, S. F., Schmidt, G. D. (+23 co-authors) 2003b, ApJ, 583, 902

Wolfe, M. et al. 2003, in prep

York, D. G., Adelman, J., Anderson, J. E. (+141 co-authors) 2000, AJ, 120, 1579 